\title{
Vortex bubble formation in pair plasmas
}

\author{
V. I. Berezhiani, ${ }^{1,2}$ N. L. Shatashvili, ${ }^{1,3}$ S. M. Mahajan, ${ }^{4}$ and B. N. Aleksić ${ }^{5,6}$ \\ ${ }^{1}$ Andronikashvili Institute of Physics, Tbilisi 0177, Georgia \\ ${ }^{2}$ School of Physics, Free University of Tbilisi, Georgia \\ ${ }^{3}$ Department of Physics, Faculty of Exact and Natural Sciences, Ivane Javakhishvili Tbilisi State University, Tbilisi 0179, Georgia \\ ${ }^{4}$ Institute for Fusion Studies, The University of Texas at Austin, Austin, Texas 78712, USA \\ ${ }^{5}$ Institute of Physics Belgrade, University of Belgrade, Belgrade, Serbia \\ ${ }^{6}$ Texas A\&M University at Qatar, Doha, Qatar \\ (Received 5 May 2013; published 18 July 2013)
}

\begin{abstract}
It is shown that delocalized vortex solitons in relativistic pair plasmas with small temperature asymmetries can be unstable for intermediate intensities of the background electromagnetic field. Instability leads to the generation of ever-expanding cavitating bubbles in which the electromagnetic fields are zero. The existence of such electromagnetic bubbles is demonstrated by qualitative arguments based on a hydrodynamic analogy, and by numerical solutions of the appropriate nonlinear Schrödinger equation with a saturating nonlinearity.
\end{abstract}

DOI: 10.1103/PhysRevE.88.015101 PACS number(s): 52.27.Ep, 52.27.Ny, 52.35.Mw, 42.65.Tg

Nonlinear dynamics of vortex solitons-structures that permeate different fields of physics, such as nonlinear optics, Bose-Einstein condensate, and plasma physics-has received considerable attention in the recent past [1]. The vortex solitons usually emerge as solutions of a generalized $(2+1) D$-dimensional nonlinear Schrödinger equation (NSE) with a local saturating nonlinearity $[2,3]$. The NSE with a defocusing (for instance, cubic) nonlinearity admits stable vortex soliton solutions. These solutions, with an angular $2 \pi$ phase ramp, and appearing as local dark minima in an otherwise bright background, are viewed as the most fundamental two-dimensional solitons embodied in the NSE. The NSE with a focusing nonlinearity also supports localized vortex solitons with phase dislocations surrounded by one or many bright rings. The latter, however, are unstable against symmetry-breaking perturbations that can cause the breakup of rings into filaments.

New and interesting behavior arises when the nonlinearity can change its character (as a function of the amplitude, for instance) from focusing to defocusing or vice versa. A representative example is a sign-changing, cubic-quintic (CQ) nonlinearity (focusing for lower and defocusing for higher intensities). The NSE with a CQ nonlinearity can, under certain conditions, support the existence of stable localized as well as delocalized vortex soliton solutions (for details see [4]). Though CQ ansatz is frequently invoked to model light beam propagation in various optical media [5] (such as non-Kerr crystals, chalcogenide glasses, semiconductors, and so on), its validity to describe these materials does require additional exploration.

In contrast to the optical media, an NSE with a focusingdefocusing nonlinearity can naturally emerge from the basic physics models when one investigates the propagation of large amplitude electromagnetic pulses in a plasma [6,7]. In some of our recent publications [8,9], we showed that the dynamics of the short intense electromagnetic pulses propagating in a relativistic pair plasma with small temperature asymmetry does, indeed, obey an NSE with, what may be called, a saturation nonlinearity. Though always positive (unlike the CQ nonlinearity), the saturation nonlinearity vanishes for high intensities, and thus combines the focusing-defocusing natures.
The particular form of the saturation function in the NSE derived in [8,9], leads to the existence of localized as well as delocalized optical vortex soliton solution (we term these solitons as LOVS and OVS, respectively). The stability of LOVS (both single and multicharged) has been investigated in detail and the stability areas were established. Although the stability of the OVS has been established for high intensities of the background fields [9], for intermediate intensities the vortex solitons can be unstable. Indeed Kim et al. [10] (see also [11]) demonstrated numerically that delocalized vortices described by NSE with CQ saturation turn out to be unstable for certain intermediate intensities leading to the formation of an ever-expanding circular ring. One is naturally faced with the question if similar behavior would pertain for the saturation nonlinearity. Unfortunately the standard linear modal stability analysis is inadequate to deal with nonexponential growth that could be associated with non-Hermitian (non-self-adjoint) linear operators. To determine the stability of the OVS in a pair plasma, therefore, we resort to a numerical solution of the NSE.

The nonlinear $(2+1) D$ evolution of an electromagnetic (EM) pulse propagating in an arbitrary pair plasma with temperature asymmetry can be described by an NSE. The dimensionless evolution equation for the slowly varying envelope of the vector potential (in comoving with group velocity frame) reads [9]

$$
i \frac{\partial A}{\partial t}+\nabla_{\perp}^{2} A+f\left(|A|^{2}\right) A=0 .
$$

The nonlinearity function

$$
f\left(|A|^{2}\right)=\frac{|A|^{2}}{\left(1+|A|^{2}\right)^{2}}
$$

has a very unusual feature: in the ultrarelativistic limit $\left(|A|^{2} \gg 1\right)$ it tends to be 0 and the nonlinear term vanishes.

Before proceeding further, we summarize the steps that led to (1). We had started with a circularly polarized EM pulse $\sim \widehat{A}(\hat{\mathbf{x}}+i \hat{\mathbf{y}}) \exp \left(i k_{0} z-\omega_{0} t\right)$ with mean frequency $\omega_{0}$ and mean wave number $k_{0}$ and where $\widehat{A}$ is the slowly varying amplitude. In Eq. (1), $\nabla_{\perp}^{2}=\partial^{2} / \partial x^{2}+\partial^{2} / \partial y^{2}$ is the diffraction operator, and various dimensionless quantities have been defined as $A=|e| \widehat{A} /\left[m G\left(T_{0}^{-}\right) c^{2}\right], r=\left(\omega_{e} / c \epsilon k^{1 / 2}\right) r$, 
$t=\left(\omega_{e}^{2} / 2 \omega_{0} \epsilon^{2} k\right) t$, where $\omega_{e}=\left(4 \pi e^{2} n_{0} / m\right)^{1 / 2}$ is the electron Langmuir frequency and $m$ is the electron mass. The charges $q^{ \pm}$and masses $m^{ \pm}$of positive and negative ions are assumed to be the same (we mainly concentrate on the specific case of an electron-positron plasma, i.e., $q^{+}=e^{+}=q^{-}=$ $-e^{-}=|e|$ and $\left.m^{+}=m^{-}=m\right)$. The equilibrium state of the system is characterized by an overall charge neutrality $n_{0}^{+}=n_{0}^{-}=n_{0}$, where $n_{0}^{+}$and $n_{0}^{-}$are the unperturbed number densities of the positive and negative species, respectively. The background temperatures of plasma species are $T_{0}^{ \pm}$ $\left(T_{0}^{+} \neq T_{0}^{-}\right)$and $m G\left(z^{ \pm}\right)=m K_{3}\left(z^{ \pm}\right) / K_{2}\left(z^{ \pm}\right)$is the "effective mass" $\left[z^{ \pm}=m c^{2} / T^{ \pm}\right]$, where $K_{v}$ are the modified Bessel functions. For the nonrelativistic temperatures $\left(T^{ \pm} \ll m c^{2}\right)$ $G^{ \pm}=1+5 T^{ \pm} / 2 m c^{2}$ and for the ultrarelativistic temperatures $\left(T^{ \pm} \gg m_{\alpha} c^{2}\right) G^{ \pm}=4 T^{ \pm} / m c^{2} \gg 1$. The parameter $\epsilon=$ $\left[G\left(T_{0}^{+}\right)-G\left(T_{0}^{-}\right)\right] / G\left(T_{0}^{+}\right)$measures the background temperature asymmetry of plasma species. For the nonrelativistic temperatures $\epsilon=5\left(T_{0}^{+}-T_{0}^{-}\right) / 2 m c^{2}$ while in ultrarelativistic case $\epsilon=\left(T_{0}^{+}-T_{0}^{-}\right) / T_{0}^{+}$. The numerical factor $\kappa=1 / 4$ for nonrelativistic temperatures $(=1 / 3$ for ultrarelativistic temperatures). In deriving Eq. (1) with Eq. (2), we have assumed that the plasma is highly transparent and the longitudinal extent of the pulse is much shorter than its transverse dimensions. The medium is self-focusing $(d f / d|A|>0)$ for $|A|<1$, while it becomes defocusing $(d f / d|A|<0)$ for higher intensities $(|A|>1)$.

To investigate the vortex soliton solutions of Eq. (1), one makes the ansatz $A=A(r) \exp (i \lambda t+i m \theta)$, where the integer $m$ defines the topological charge, and $\lambda$ is the nonlinear frequency shift. In polar coordinates, this substitution converts Eq. (1) into an ordinary differential equation. For a nonzero topological charge, the solution has a node at the origin: $A_{r \rightarrow 0} \rightarrow r^{|m|} A_{0}$, where $A_{0}$ is a constant that measures the slope of $A$ at the origin. Depending on the values of $\lambda$ and $A_{0}$ the soliton solution is either localized $A_{r \rightarrow \infty} \rightarrow 0$ or delocalized $A_{r \rightarrow \infty} \rightarrow A_{\infty}$ [where $\lambda=f\left(A_{\infty}\right)$ ]. The delocalized vortex solitons are found to exist only in the range $0<\lambda<0.25\left(\infty>A_{\infty}>1\right)$ for any value of the topological charge $m$. Thus, while near the vortex core the medium is focusing, the background intensity of the soliton $A_{\infty}>1$, i.e., far beyond the vortex core, the medium is defocusing and consequently the background field is modulationally stable.

In what follows we consider the stability of OVS. It is well known that single charge $(m=1)$ OVSs are stable in self-defocusing media. The multicharged vortices, however, despite their very long life [12], should eventually decay into $m=1$ vortices [1]. The last statement (which is based on general topological consideration) should be valid for any kind of media and will not be addressed here. For the particular system described by Eq. (1), OVSs are stable against azimuthal perturbation for any values of the allowed background field [9]; the radial stability, on the other hand, was established just for high intensities. Based on the results obtained in [10] one should expect that for the intermediate intensities of the EM field OVSs could be unstable for our mixed-type nonlinearity.

To augment our expectation it is useful to employ the hydrodynamic analogy based on so-called Madelung transformation $A=\rho^{1 / 2} \exp (i \phi)$, where $\rho=|A|^{2}$ and $\phi$ is the real phase [13]. With simple algebra (and changing a time coordinate as $t \rightarrow t / 2$ ), we can transform the NSE (1) into the following set of two coupled equations:

$$
\begin{gathered}
\frac{\partial \rho}{\partial t}+\nabla \cdot(\rho \mathbf{V})=0 \\
\frac{\partial}{\partial t}\left(\rho V_{i}\right)+\frac{\partial}{\partial x_{k}}\left(\rho V_{i} V_{k}\right)=\frac{\partial \sigma_{i k}}{\partial x_{k}} .
\end{gathered}
$$

These equations mimic, respectively, the conservation of mass and momentum for a compressible fluid of density $\rho\left(=|A|^{2}\right)$ and velocity $\mathbf{V}=\nabla \phi$; an effective stress tensor is given by

$$
\sigma_{i k}=-P(\rho) \delta_{i k}+\frac{\rho}{4} \frac{\partial^{2}(\ln \rho)}{\partial x_{i} \partial x_{k}},
$$

where $P=-\frac{1}{2} \int \rho f^{\prime}(\rho) d \rho$ is the standard hydrostatic pressure, while the second term on the right-hand side of Eq. (5) represents the quantum stress tensor. Thus, the Madelung fluid obeys an Euler-like equation of motion which, due to the presence of the quantum stress term, is drastically different from the equations describing the dynamics of an ideal fluid. However, in certain cases when-as for instance, for slowly varying perturbations-the quantum stress can be ignored and even though the obtained ideal fluid Euler
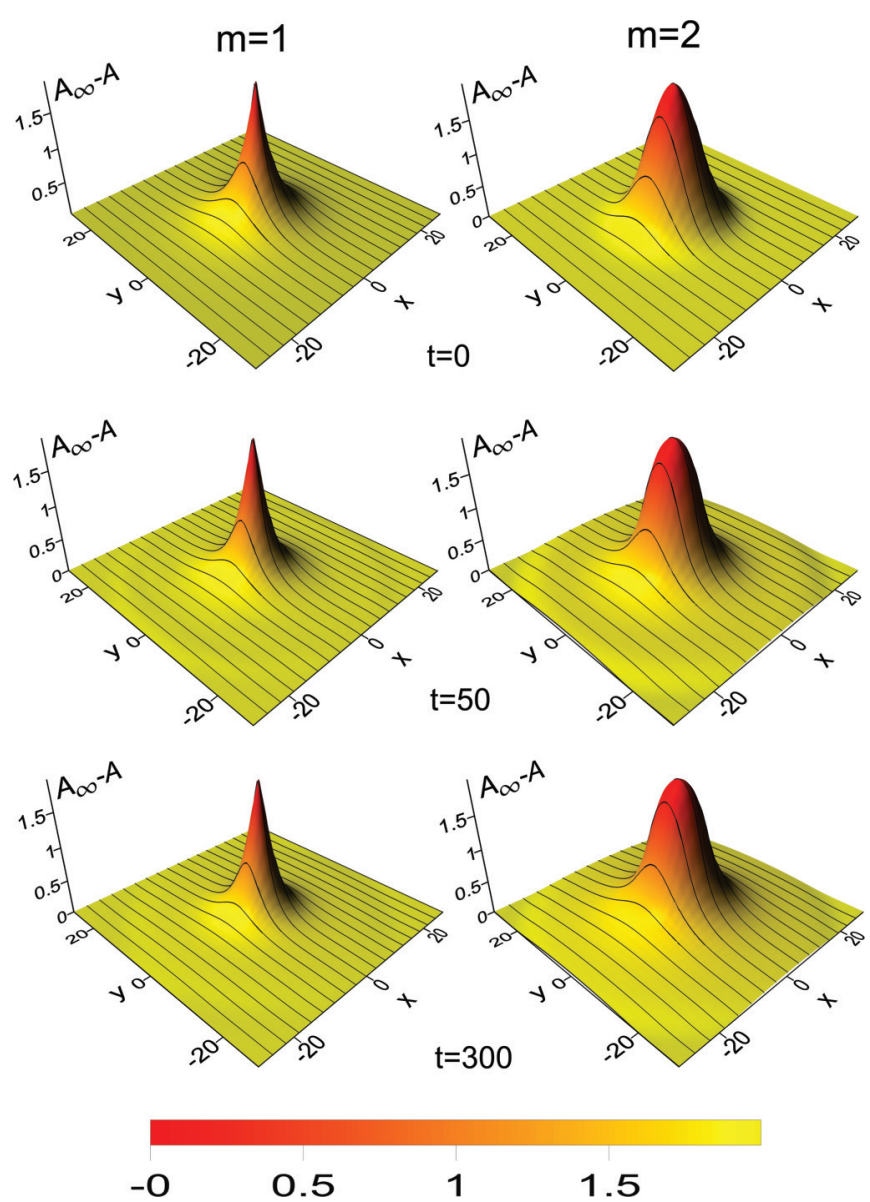

FIG. 1. (Color online) Dynamics of initial vortices with $A_{\infty}=2$ $(P>0)$ for different $t=0, t=50$, and $t=300$ time moments. Left column corresponds to the $m=1$ and right column - to the $m=2$ cases, respectively. Vertical axis corresponds to $\left[-\left(A-A_{\infty}\right)\right]$, thus, spatial patterns are presented inside out for a clear view. 

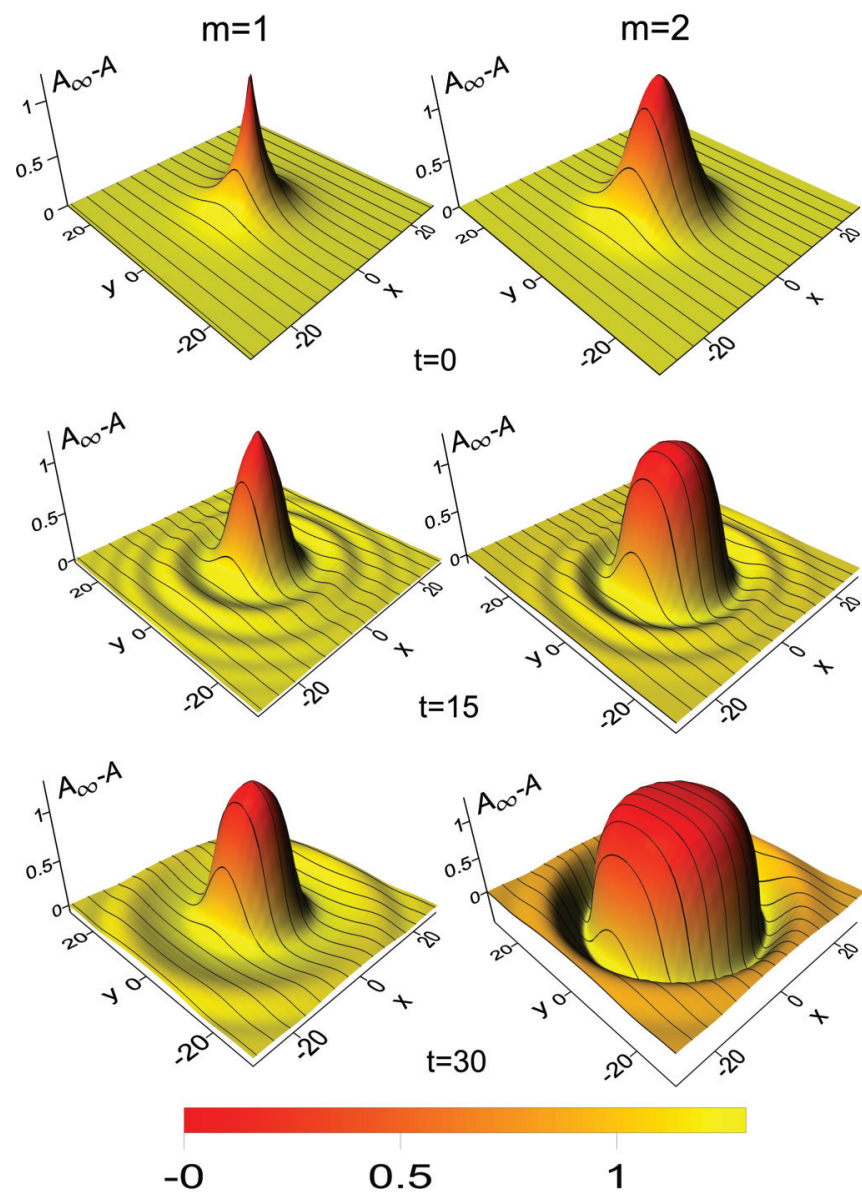

FIG. 2. (Color online) Dynamics of initial vortices with $A_{\infty}=1.3$ $(P<0)$ for different $t=0, t=15$, and $t=30$ time moments. Left column corresponds to the $m=1$ and right column- to the $m=2$ cases, respectively.

equations are not entirely physically realistic, it is found to have some important qualitative features that are helpful in understanding the complex dynamics of the problems described by NSE.

Equations (1)-(5) are valid for an arbitrary nonlinearity function $f$. For our particular case of $f=\rho /(1+\rho)^{2}$, the equivalent hydrostatic pressure may be written as

$$
P(\rho)=\ln (1+\rho)^{1 / 2}-\frac{\rho(1+2 \rho)}{(1+\rho)^{2}} .
$$

Notice that, for this pressure, the sound speed $c_{s}=$ $\sqrt{d P / d \rho}=\sqrt{0.5 \rho(\rho-1) /(1+\rho)^{3}}$ "exists" only if $\rho>1$. It should be pointed out that the above hydrostatic pressure is positive for high densities and becomes negative for $\rho<2.16$. In classical hydrodynamics [14] negative pressures are often associated with cavitation, which involves the formation of topological defects in the form of bubbles. For the OVS the density $\rho=0$ at $r=0$, and tends to its equilibrium value $\rho_{r \rightarrow \infty} \rightarrow \rho_{0}>1\left(\rho_{0}=A_{\infty}^{2}\right)$. Thus beyond the vortex core, the sound can propagate, i.e., the background is modulationally stable. The radial velocity of the fluid is zero while it performs a differential rotation with azimuthal velocity $V_{\theta}=m / r$. If $1<\rho_{0}<2.16$, the hydrostatic pressure becomes negative beyond the vortex core and the negative pressure forces may no longer be balanced by the centrifugal and quantum pressure forces. Thus, we expect that for the certain class of perturbation the cavitation could take place, and since $\rho(0)$ remains zero due to the topological constraints (strictly speaking for $m=1$ ) the cavitating bubble expanding in the radial direction, might be formed.

In order to verify the phenomenon suggested by the analysis of the Madelung-fluid "translation" of the NSE, we have carried out the stability analysis by solving Eq. (1) numerically. The dynamics of initial OVS-like singly and doubly charged field distribution was followed for negative $P\left(\rho_{0}\right)<0\left(1<A_{\infty}^{2}<2.16\right)$ as well as positive pressures $P\left(\rho_{0}\right)>0\left(A_{\infty}^{2}>2.16\right)$. For positive pressure (Fig. 1), the initial field distributions evolve toward the stationary OVS with parameters determined by $A_{\infty}$ (plotted spatial patterns are presented inside out for a clear view). For negative pressure (Fig. 2), on the other hand, no OVS are formed; instead, the vortex core expands out constantly in time leading to the generation of ever-expanding electromagnetic bubbles.

We have shown that the delocalized vortex solitons associated with an NSE, containing the particular form of saturating nonlinearity arising naturally in a relativistic pair plasma with temperature "asymmetry," can become unstable for moderately intense background fields leading to the formation of an ever-expanding core. Though the changes in the core plasma density and temperature are small, the electromagnetic fields are expelled from within the expanding core. The formation of such electromagnetic bubbles could have rather interesting implications for structure formation both in cosmic and astrophysical settings, and for pair plasmas that may soon be available in laboratory conditions.

The authors acknowledge a special debt to the Abdus Salam International Centre for Theoretical Physics, Trieste, Italy; the authors also thank Dr. Milivoj Belić for valuable discussions. The work of V.I.B. and N.L.S. was partially supported by Shota Rustaveli NSF project 1-4/16 (Grant No. GNSF/ST09305-4-140). The work of S.M.M. was supported by USDOE Contract No. DE-FG 03-96ER-54366. The work of B.N.A. was partially supported by the Qatar NRF project NPRP 5674-1-114 and by the Ministry of Science of Serbia under the project OI 171006.
[1] A. S. Desyatnikov and Y. S. Kivshar, Prog. Opt. 47, 291 (2005).

[2] F. Vidal and T. W. Johnston, Phys. Rev. Lett. 77, 1282 (1996); Phys. Rev. E 55, 3571 (1997).

[3] W. J. Firth and D. V. Skryabin, Phys. Rev. Lett. 79, 2450 (1997).
[4] V. I. Berezhiani, V. Skarka, and N. B. Aleksić, Phys. Rev. E 64, 057601 (2001).

[5] B. L. Lawrence and G. I. Stegeman, Opt. Lett. 23, 591 (1998); G. Boudebs, S. Cherukulappurath, H. Leblond, J. Troles, 
F. Smektala, and F. Sanchez, Opt. Commun. 219, 427 (2003); V. Skarka, N. B. Aleksić, M. Derbazi, and V. I. Berezhiani, Phys. Rev. B 81, 035202 (2010).

[6] P. K. Shukla, N. N. Rao, M. Y. Yu, and N. L. Tsintsadze, Phys. Rep. 138, 1 (1986); V. I. Berezhiani, S. M. Mahajan, Z. Yoshida, and M. Ohhashi, Phys. Rev. E 65, 047402 (2002); V. I. Berezhiani and S. M. Mahajan, Phys. Rev. Lett. 73, 1110 (1994); T. Tatsuno, V. I. Berezhiani, and S. M. Mahajan, Phys. Rev. E 63, 046403 (2001); S. Kartal, L. N. Tsintsadze, and V. I. Berezhiani, ibid. 53, 4225 (1996).

[7] A. Vincotte and L. Bergé, Phys. Rev. Lett. 95, 193901 (2005); Physica D 223, 163 (2006); V. Skarka, N. B. Aleksić, and V. I. Berezhiani, Phys. Lett. A 319, 317 (2003).
[8] S. M. Mahajan, N. L. Shatashvili, and V. I. Berezhiani, Phys. Rev. E 80, 066404 (2009).

[9] V. I. Berezhiani, S. M. Mahajan, and N. L. Shatashvili, Phys. Rev. A 81, 053812 (2010); J. Plasma Phys. 76, 467 (2010).

[10] W. Kim, S. Chae, and H. T. Moon, Phys. Lett. A 276, 91 (2000).

[11] N. G. Berloff, Fluid Dyn. Res. 41, 051403 (2009).

[12] I. Aranson and V. Steinberg, Phys. Rev. B 53, 75 (1996).

[13] T. Takabayasi, Prog. Theor. Phys. 8, 143 (1952); C. Josserand, Y. Pomeau, and S. Rica, Phys. Rev. Lett. 75, 3150 (1995); D. Novoa, H. Michinel, and D. Tommasini, ibid. 103, 023903 (2009).

[14] G. Batchelor, An Introduction to Fluid Mechanics (Cambridge University Press, Cambridge, 1967). 